\title{
OPEN Acrolein contributes to human colorectal tumorigenesis through the activation of RAS-MAPK pathway
}

\author{
Hong-Chieh Tsai ${ }^{1,2}$, Han-Hsing Tsou $3,4,5$, Chun-Chi Lin ${ }^{6,7,8}$, Shao-Chen Chen ${ }^{3,4}$, \\ Hsiao-Wei Cheng ${ }^{9,10}$, Tsung-Yun Liu ${ }^{3,4}$, Wei-Shone Chen ${ }^{6,7,8}$, Jeng-Kai Jiang ${ }^{6,7,8}$, \\ Shung-Haur Yang ${ }^{6,7,8,11}$, Shih-Ching Chang ${ }^{6,7,8}$, Hao-Wei Teng ${ }^{6,7,12 \bowtie}$ \& Hsiang-Tsui Wang ${ }^{9,10 \bowtie ~}$
}

Colorectal cancer (CRC) is one of the most well-known malignancies with high prevalence and poor 5-year survival. Previous studies have demonstrated that a high-fat diet (HFD) is capable of increasing the odds of developing CRC. Acrolein, an IARC group 2A carcinogen, can be formed from carbohydrates, vegetable oils, animal fats, and amino acids through the Maillard reaction during the preparation of foods. Consequently, humans are at risk of acrolein exposure through the consumption of foods rich in fat. However, whether acrolein contributes to HFD-induced CRC has not been determined. In this study, we found that acrolein induced oncogenic transformation, including faster cell cycling, proliferation, soft agar formation, sphere formation and cell migration, in NIH/3T3 cells. Using xenograft tumorigenicity assays, the acrolein-transformed NIH/3T3 clone formed tumors. In addition, CDNA microarray and bioinformatics studies by Ingenuity Pathway Analysis pointed to the fact that RAS/MAPK pathway was activated in acrolein-transformed clones that contributed to colon tumorigenesis. Furthermore, acrolein-induced DNA damages (Acr-dG adducts) were higher in CRC tumor tissues than in normal epithelial cells in CRC patients. Notably, CRC patients with higher levels of Acr-dG adducts appeared to have better prognosis. The results of this study demonstrate for the first time that acrolein is important in oncogenic transformation through activation of the RAS/MAPK signaling pathway, contributing to colon tumorigenesis.

Colorectal cancer (CRC) is the third most frequent neoplasm worldwide (https://www.wcrf.org). Although diagnosis and therapy have advanced significantly over the past 10 years, its prevalence is rising, and the 5-year survival rate is poor ${ }^{1}$. CRC has become a significant issue for healthcare in Asian countries, with a two to fourfold increase in incidence over the rate decades ago. CRC emerges from benign neoplasms and develops into adenocarcinomas through a stepwise histological progression sequence, continuing from either adenomas or hyperplastic polyps/serrated adenomas. Genetic modifications have been related to specific steps in this adenoma-carcinoma sequence and are believed to drive the histological progression of $\mathrm{CRC}^{2,3}$. CRC arises from an association of genetic and environmental factors, and it has been identified as involving multiple cell signaling pathways, such as Wnt, epidermal growth factor receptor/mitogen-activated protein kinase (EGFR/MAPK), tumor protein 53 (TP53), phosphoinositide 3-kinase (PI3K), and transforming growth factor beta (TGF $\beta$ )/ $\mathrm{SMAD}^{4,5}$. Epidemiologic studies have shown that lifestyle and dietary habits impact the danger of developing

\footnotetext{
${ }^{1}$ Department of Neurosurgery, Linkou Chang Gung Memorial Hospital, Taoyuan 333, Taiwan. ${ }^{2}$ School of Traditional Chinese Medicine, Chang Gung University, Taoyuan 333, Taiwan. ${ }^{3}$ Institute of Food Safety and Health Risk Assessment, National Yang-Ming University, Taipei, Taiwan. ${ }^{4}$ Institute of Food Safety and Health Risk Assessment, National Yang Ming Chiao Tung University, Taipei, Taiwan. ${ }^{5}$ Kim Forest Enterprise Co., Ltd., Taipei, Taiwan. ${ }^{6}$ School of Medicine, National Yang-Ming University, Taipei, Taiwan. ${ }^{7}$ School of Medicine, National Yang Ming Chiao Tung University, Taipei, Taiwan. ${ }^{8}$ Division of Colon and Rectum Surgery, Department of Surgery, Taipei Veterans General Hospital, Taipei, Taiwan. ${ }^{9}$ Institute of Pharmacology, College of Medicine, National Yang-Ming University, Taipei, Taiwan. ${ }^{10}$ Institute of Pharmacology, College of Medicine, National Yang Ming Chiao Tung University, Taipei, Taiwan. ${ }^{11}$ Department of Surgery, National Yang Ming Chiao Tung University Hospital, Yilan, Taiwan. ${ }^{12}$ Division of Medical Oncology, Department of Oncology, Taipei Veterans General Hospital, Taipei, Taiwan. ${ }^{\square}$ email: hwteng1971@gmail.com; htwang01@ym.edu.tw
} 
$\mathrm{CRC}^{6}$. In particular, the intake of foods rich in fat and with low fiber content (known as a high-fat diet (HFD) or Western-style diet) increases the odds of developing $\mathrm{CRC}^{7-9}$.

Acrolein (2-propenal), the most reactive $\alpha, \beta$-unsaturated aldehyde, is a highly mutagenic and highly oxidizing environmental toxin ${ }^{10}$. The most well-studied source of acrolein exposure is tobacco smoking, which has been shown to be associated with oral, lung and bladder cancer ${ }^{11-16}$. IARC working groups re-evaluated acrolein as probably carcinogenic to humans (Group 2A) on the basis of sufficient evidence of carcinogenicity in experimental animals and strong mechanistic evidence ${ }^{17}$. Nonetheless, its dietary exposure and consequences are under investigation. Acrolein can be formed from carbohydrates, vegetable oils, animal fats, and amino acids during the preparation of foods ${ }^{18}$. It is formed during the Maillard reaction as a result of the conversion of amino acids $^{19,20}$ and the oxidative deamination of polyamines ${ }^{21}$. Likewise, acrolein can be detected in the emissions of varieties of heated or overheated cooking oils and as such is found abundantly in fried food such as French fries $^{22}$. Additionally, recent reports demonstrated that gut microbial glycerol metabolism prompts the formation of reuterin, which is an additional source of endogenous acrolein ${ }^{23}$. Reuterin is an antimicrobial multicomponent system comprising 3-hydroxypropionaldehyde, its dimer and hydrate, and acrolein. Our recent studies have shown that exposure to acrolein following consumption of fried food influences local oral cavity homeostasis ${ }^{24}$. Consequently, humans are in danger of acrolein exposure through consumption of food rich in high fat ${ }^{25,26}$.

Although the association between HFD and CRC risk has been known for quite a while ${ }^{27-29}$, the exact mechanisms underlying HFD-induced colon cancer risk and recurrence have remained unclear. The large-scale connections of dietary components with one another and with metabolism make it hard to specifically recognize the components in HFD that might cause $\mathrm{CRC}^{8}$. Since acrolein can be produced during the preparation of foods ${ }^{18}$, we aimed to investigate the role of acrolein in CRC tumorigenesis. In the present study, we determined the effect of acrolein on oncogenic transformation using NIH/3T3 cells with xenograft tumorigenesis mouse models. Furthermore, cDNA microarray analysis with Ingenuity Pathway Analysis (IPA) was performed in acrolein-transformed NIH/3T3 cells. Finally, acrolein-induced DNA damages (Acr-dG adducts) were analyzed in tumor tissues and normal epithelial tissues of CRC patients, and the levels of Acr-dG adducts were associated with tumor characteristics and CRC patient survival.

\section{Results}

Acrolein treatment induced cell proliferation, anchorage-independent activity, spheroid formation ability and cell migration capacity. To determine the potential role of acrolein in oncogenic transformation, we treated NIH/3T3 cells with a low dose of acrolein $\left(7.5 \mu \mathrm{M}, \mathrm{IC}_{10}\right)$ for one month and selected NIH/3T3 Acr clones \#1-\#7 (Supplementary Fig. 1A). The soft agar colony formation activity of these seven clones was analyzed, and the results showed that NIH/3T3 Acr-clones \#3, \#4 and \#6 formed more colonies than the others (Supplementary Fig. 1B). Cell proliferation analysis showed that NIH/3T3 Acr-clone \#4 (doubling time $=31.0 \mathrm{~h}$ ) had faster proliferation than parental cells (doubling time $=39.4 \mathrm{~h}$ ); however, NIH/3T3 Acr-clone \#3 (doubling time $=55.0 \mathrm{~h}$ ) or NIH/3T3 Acr-clone\#6 (doubling time $=42.9 \mathrm{~h}$ ) showed the opposite phenomenon (Fig. 1A, Supplementary Fig. 1C). Therefore, we selected NIH/3T3Acr-clone \#4 for subsequent analysis. Consistently, cell cycle analysis showed that the ratio of NIH/3T3 Acr-clone\#4 cells in S phase was markedly higher than that in parental cells (Fig. 1B, Supplementary Fig. 1D), indicating that acrolein promotes S-phase DNA synthesis and accelerates cell proliferation. Anchorage-independent activity (Fig. 1C) in NIH/3T3 Acr-clone\#4 cells was also increased compared to parental NIH/3T3 cells using a soft agar colony formation assay. Spheroid formation ability on ultralow attachment plates of NIH/3T3 Acr-clone\#4 was also enhanced (Fig. 1D). In addition, NIH/3T3 Acr-clone\#4 cells showed enhanced migration capacity compared with NIH/3T3-mock cells (Fig. 1E) using a Transwell assay. However, the drug sensitivity of NIH/3T3 Acr-clone\# 4 toward chemotherapeutic agents such as oxaliplatin and 5-FU was similar to that of parental NIH/3T3 cells (Supplementary Fig. 2). These results suggest that acrolein increases the cell cycle rate, proliferation, colony formation activity, spheroid formation ability and cell migration capacity.

NIH/3T3 Acr-clone\#4 formed tumors in xenograft nude mice. Our in vitro results indicate that acrolein can transform normal mouse NIH/3T3 fibroblasts into malignant cells. To confirm its tumorigenic potential, we performed in vivo studies of tumor xenografts in nude mice using parental NIH/3T3 cells as the negative control. NIH/3T3 Acr-clone\#4 and parental NIH/3T3 cells were injected subcutaneously into the right axillary fossa $\left(5 \times 10^{6}\right.$ cells/animal). Three weeks after injecting NIH/3T3 Acr-clone\#4 into nude mice, nodular neoplasms could be observed, while tumors were obvious at 10 days, whereas the parental NIH/3T3 cells failed to form any tumors (Fig. 2A). Tumors formed by NIH/3T3 Acr-clone\#4 cells were observed, and their volumes and growth curves were calculated for 4 weeks after the tumors could be observed (Fig. 2B,C). These data further indicate that acrolein leads to oncogenic transformation in vivo.

Acrolein induced the RAS/MAPK signaling pathway in CRC tumorigenesis using ingenuity pathway analysis (IPA). To determine the underlying mechanisms by which acrolein induced oncogenic transformation, cDNA microarray analysis with IPA was performed in NIH/3T3 Acr-clone\#4 cells (Fig. 3A). The results showed that four genes (Rnd1, Rras2, myc and PI3Kcb) involved in the RAS/MAPK signaling pathway were upregulated in acrolein-transformed clone \#4 (NIH/3T3 Acr-clone \#4) (Fig. 3B, Supplementary Table 1). These results were confirmed using Western blot analysis (Fig. 3C, Supplementary Fig. 3A). Furthermore, we also found that acrolein activated the RAS/MAPK signaling pathway and increased c-myc in NIH/3T3 cells and the human normal colon epithelium CCD-841CoN (Fig. 3D,E, Supplementary Fig. 3B,C). Intriguingly, acrolein induced cell proliferation, colony formation activity and cell migration capacity in CCD-841CoN cells (Fig. 4A,D). Activation of the RAS/MAPK signaling pathway was also observed in the CCD-841CoN Acr clone 
A

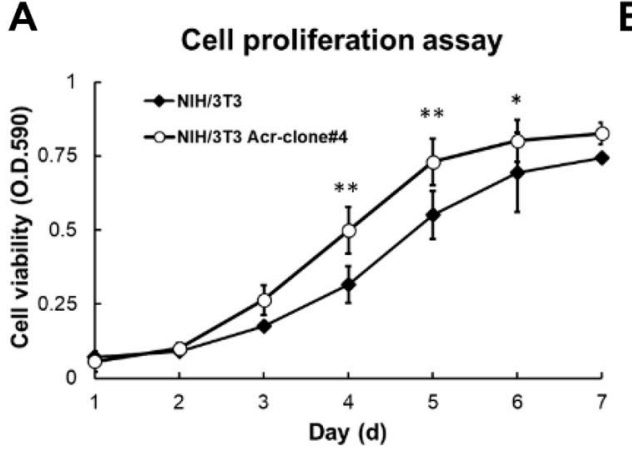

C

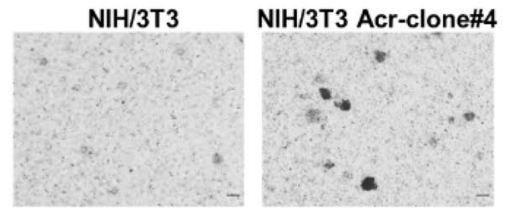

Soft agar colony formation assay

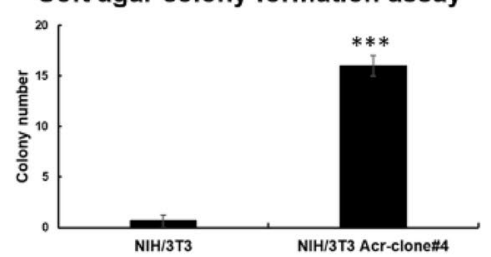

B

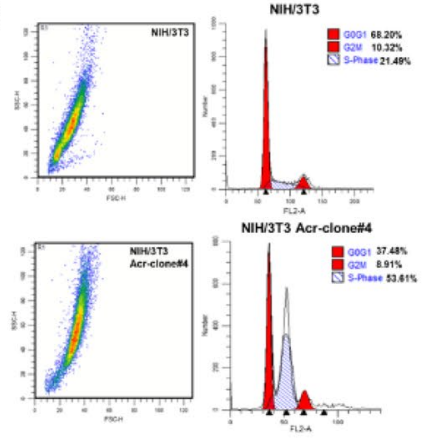

D

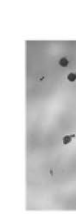

NIH/3T3
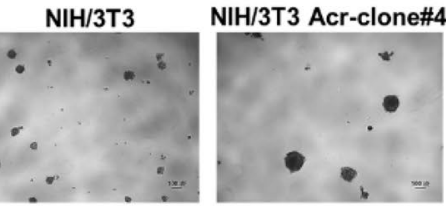

Sphere formation assay

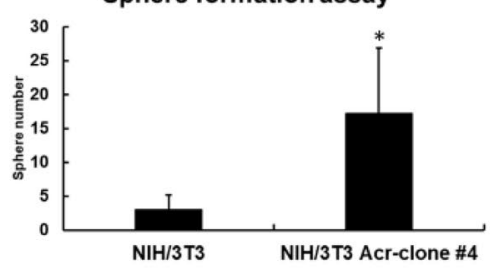

Cell cycle analysis

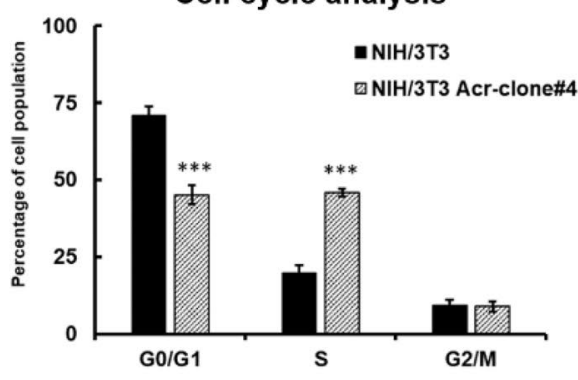

E

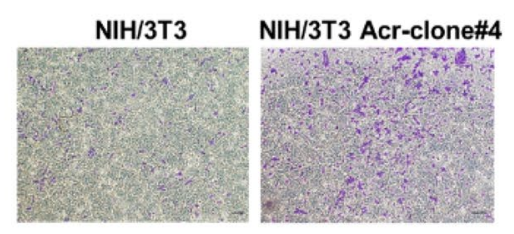

Migration assay

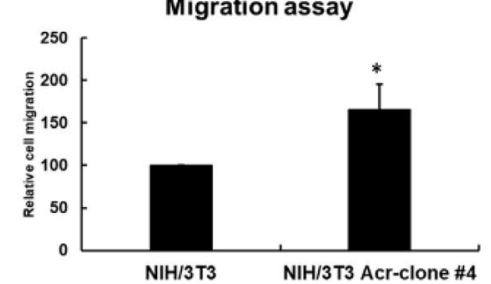

Figure 1. Acrolein induced oncogenic transformation using a cellular model of NIH/3T3 cells. NIH/3T3 cells were treated with acrolein $($ Acr, $7.5 \mu \mathrm{M})$ for one month and named NIH/3T3 Acr-clone\#. (A) Cell proliferation of NIH/3T3 Acr-clone \#4 cells was analyzed using MTT assays. (B) Cell cycle progression of NIH/3T3 Acr-clone \#4 cells was analyzed using cell cycle analysis with PI staining. (C) Soft agar anchorage-dependent cell growth of NIH/3T3 Acr-clone \#4 was analyzed using a soft agar assay. (D) The spheroid formation ability of NIH/3T3 Acr-clone \#4 cells was analyzed on ultralow attachment plates. (E) The cell migration activity of NIH/3T3 Acrclone \# 4 cells was analyzed using Transwell migration analysis. Scale bar: $100 \mu \mathrm{m}$. NIH/3T3 Acr-clone \#4 had the highest cell transformation activity. Student's $t$ tests were used to determine statistical significance, and twotailed $\mathrm{p}$-values are shown. ${ }^{\star} \mathrm{p}<0.05,{ }^{* *} \mathrm{p}<0.01,{ }^{* * *} \mathrm{p}<0.005$ compared with NIH/3T3 parental cells.

(Fig. 4E, Supplementary Fig. 3D) as well as human colon cancer cell lines, SW480 and HCT116 (Supplementary Fig. 4). These results indicate that acrolein induced oncogenic transformation through activation of the RAS/ MAPK pathways.

Immunohistochemistry analysis of acrolein-DNA (Acr-dG) levels in human colon cancers. Acrolein can react with DNA-inducing modifications, which, if not repaired, can result in mutations and lead to cancer development. Acrolein has been shown to produce propano-2'-deoxyguanosine (Acr-dG) adducts in human cells ${ }^{15,30,31}$. Acr-dG adducts are mutagenic and induce mainly $\mathrm{G}$ to $\mathrm{T}$ and $\mathrm{G}$ to $\mathrm{A}$ mutations ${ }^{15,31-40}$. To further investigate whether acrolein contributes to colon cancer formation, we analyzed Acr-dG adduct expression in CRC tissues and normal epithelial cells adjacent to tumor tissues using immunohistochemical (IHC) staining. The results showed that Acr-dG adduct levels were mainly located in the nucleus and were higher in CRC tumor tissue than in normal epithelial cells in 18 CRC patients (Fig. 5A-C). Based on our cDNA microarray data, c-myc was upregulated in acrolein-transformed cell clones (Fig. 3C). We further analyzed c-myc levels in CRC tissues and normal epithelial cells in the same patients using IHC staining (Supplementary Fig. 5). Similar to Acr-dG adduct levels, higher c-myc levels were observed in CRC tumor tissues than in normal epithelial cells.

Higher Acr-dG expression is associated with improved survival in CRC patients. We further evaluated the effect of Acr-dG expression on CRC characteristics and patient survival. The demographic data are shown in Table 1. Of 236 CRC patients, the majority had adenocarcinoma (211/236, 89.4\%), and advanced stage III and IV cancer was diagnosed in 64\% (151/236) of patients. The expression of Acr-dG was defined as low (H score $<50)$ or high $(\mathrm{H}$ score $\geq 50)$ based on the results showing Acr-dG levels in CRC tumor tissues and normal epithelia (Fig. 5B,C). Kaplan-Meier survival analysis revealed that the median survival was 103.4 months for CRC patients with high Acr-dG levels and 74.3 months for CRC patients with low Acr-dG levels, indicating that high expression of Acr-dG in tumor tissue was associated with better CRC patient overall survival $(\mathrm{p}=0.0033$, Fig. 5D). In addition, high expression of Acr-dG was inversely correlated with clinical stages and grades using chi-square analysis (Table 1). The impact of confounders on Acr-dG was analyzed using Cox proportional haz- 
A

NIH/3T3

\section{$\mathrm{NIH} / 3 \mathrm{T3}$ Acr-clone \#4}
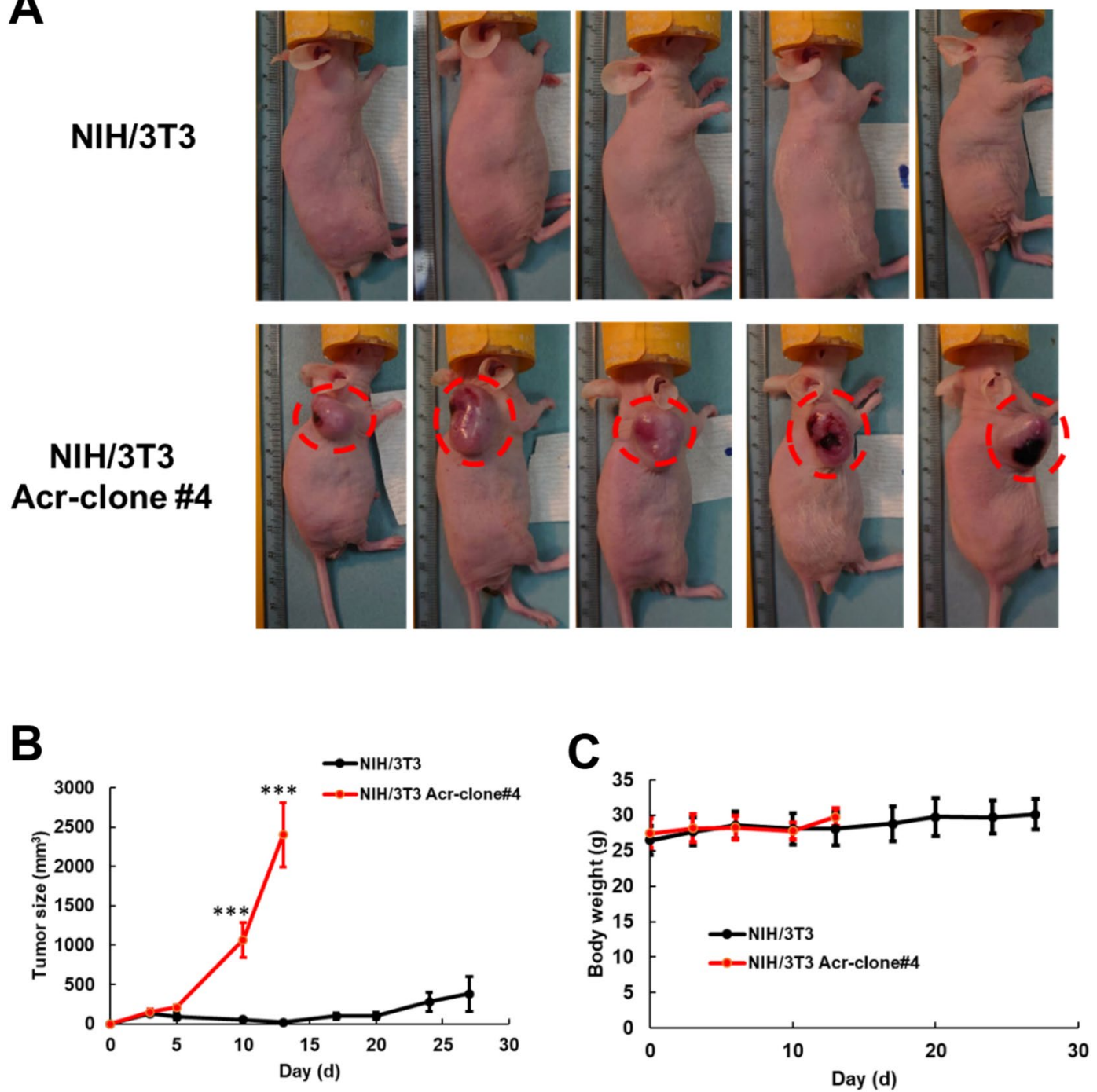

Figure 2. Xenograft mouse model of acrolein-transformed clones. (A) Overall view of tumors formed by acrolein-transformed NIH/3T3 cell clone (Acr-clone\#4). Tumors in nude mice were observed after injection with acrolein-transformed clone \#4, whereas none were observed after injection with mouse fibroblasts or NIH/3T3 cells. (B) Tumor growth curves and (C) body weight of nude mice in different experimental groups $(\mathrm{n}=5)$. Student's $t$ tests were used to determine statistical significance, and two-tailed $\mathrm{p}$-values are shown. ${ }^{* * *} \mathrm{p}<0.005$ compared with NIH/3T3 parental cells.

ards models, as shown in Supplementary Tables 2-3. Before the multiple Cox regression analysis (Supplementary Table 2), the proportional hazards assumption test for a Cox regression model was performed (Supplementary Table 3). The Schoenfeld residuals are shown for our multivariable Cox regression model fit to the dataset with confounders (age, location, sex, acrolein, stage, grade and LVSI), as shown in Supplementary Table 4. Based on these results, Acr-dG was not an independent prognostic factor after controlling for age, location, sex, acrolein, stage, grade and LVSI. Taken together, our data suggest that CRC patients with higher Acr-dG expression in tumor tissues have a better prognosis.

\section{Discussion}

Acrolein is the most reactive $\alpha, \beta$-unsaturated aldehyde present in tobacco smoke, in ambient air pollution, and in some cooking oils heated to a high temperature ${ }^{18}$. Acrolein was previously evaluated as a group 3 carcinogen by the IARC Working Group in 1995; however, it was re-evaluated as probably carcinogenic to humans (Group 2A) ${ }^{17}$. Our previous studies have also supported that acrolein is associated with oral, lung and bladder cancer ${ }^{12,13,15,16}$. Furthermore, our current studies have shown that individuals could be exposed to acrolein from consuming fried food ${ }^{24}$. Although the association between HFD and CRC risk has been known for quite a while ${ }^{27-29}$, the exact mechanisms underlying HFD-induced colon cancer risk and recurrence remain unclear. In the present study, our results showed that acrolein induced oncogenic transformation in NIH/3T3 cells in vitro and in vivo. The underlying mechanism was through activation of the RAS/MAPK pathway, which contributes to 
A

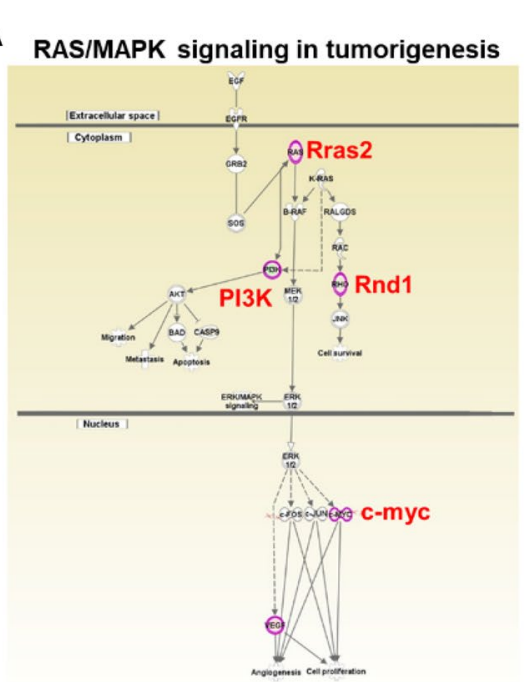

B

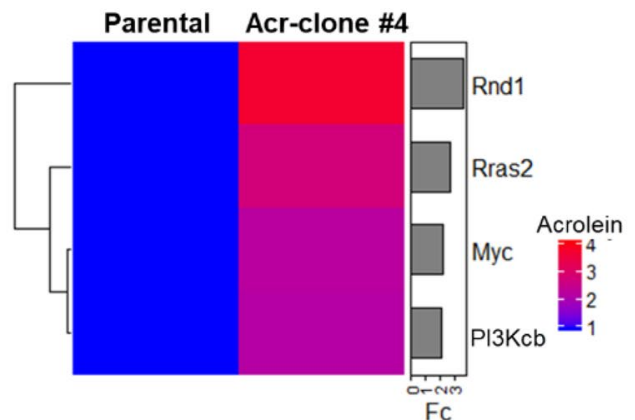

Ras

p-AKT

AKT

p-Erk

Erk

Cyclin D1

c-Myc

GAPDH

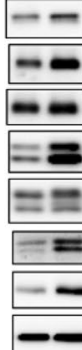

D Acrolein $(5 \mu \mathrm{M}, \mathrm{h})$ Acrolein $(\mu \mathrm{M}, 24 \mathrm{~h})$

NIH/3T3

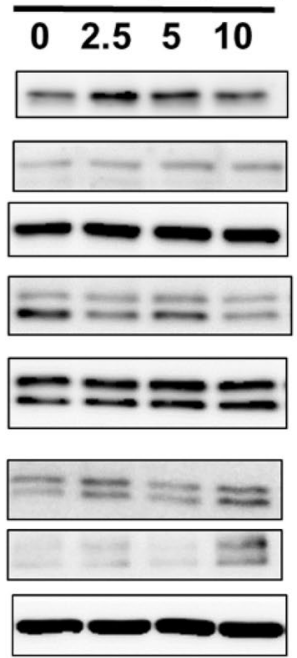

E

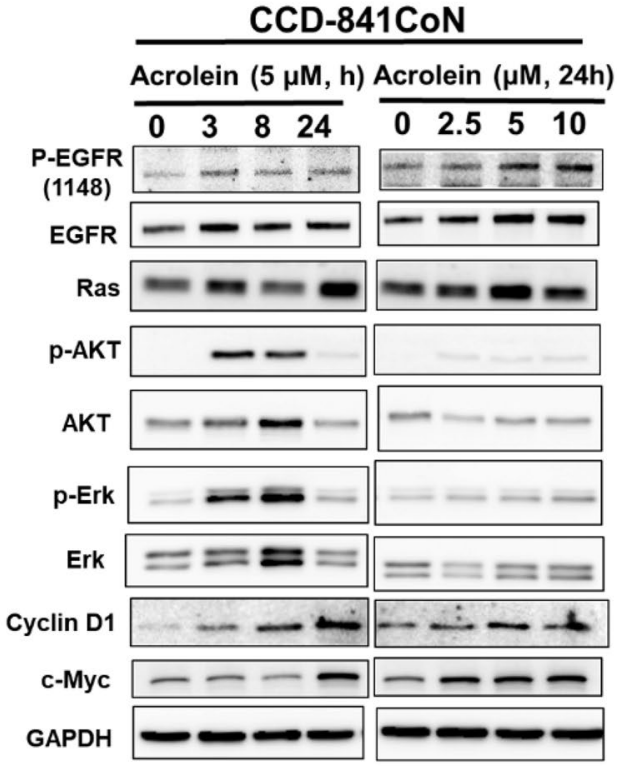

Figure 3. Ingenuity Pathway Analysis (IPA) of gene expression profiles in acrolein-transformed clones. (A) Bioinformatic analysis of the differentially expressed genes (DEGs, fold change $\geq 2$ ) was performed using IPA, and canonical pathway analysis for gene expression profiles in acrolein-transformed clone \#4 (Acr-clone \#4) is shown. (B) Heatmap of 4 genes (Rnd1, Rras2, Myc and PI3Kcb) in acrolein-transformed clone \# 4 compared to parental NIH/3T3 cells. FC, fold-change, indicates RNA expression fold change between NIH/3T3 Acr-clone\#4 and parental cells. (C) Western blot analysis of the RAS/ERK and AKT pathways in acrolein-transformed clone \#4 compared to parental NIH/3T3 cells. (D,E) Dose and time effects of acrolein on RAS expression, AKT activation, ERK activation, cyclin D1 and c-myc expression in NIH/3T3 cells (D) and CCD-841CoN cells (E) were analyzed using Western blot analysis. For dose and time effects, cells were treated with different concentrations of acrolein $(0-10 \mu \mathrm{M})$ for $24 \mathrm{~h}$ or acrolein $(5 \mu \mathrm{M})$ for 3-24 h, respectively. Original Western blots of (C-E) are shown in Supplementary Fig. 3A-C.

colon carcinogenesis. Additionally, Acr-dG adducts were higher in CRC tumor tissues than in normal epithelial cells in CRC patients. These results suggest that acrolein may contribute to colon tumorigenesis. Furthermore, slot blot analysis showed increased Acr-dG levels in mouse colon tissues fed a HFD for 24 weeks compared to mice fed a normal diet (Supplementary Fig. 6). Furthermore, we found that acrolein-protein conjugates (AcrPC) were increased in colon tissues of mice fed a HFD for 4-24 weeks (Supplementary Fig. 7), and activation of RAS/MAPK signaling was also observed in colon tissues of mice fed a HFD (data not shown). Acrolein contains a carbonyl group and an olefinic double bond and has been shown to react with cysteine, histidine, and lysine residues of proteins and with nucleophilic sites in DNA ${ }^{41-43}$. These results indicate that HFD induced acrolein production in the mouse colon and that acrolein may contribute to HFD-induced colon tumorigenesis. 


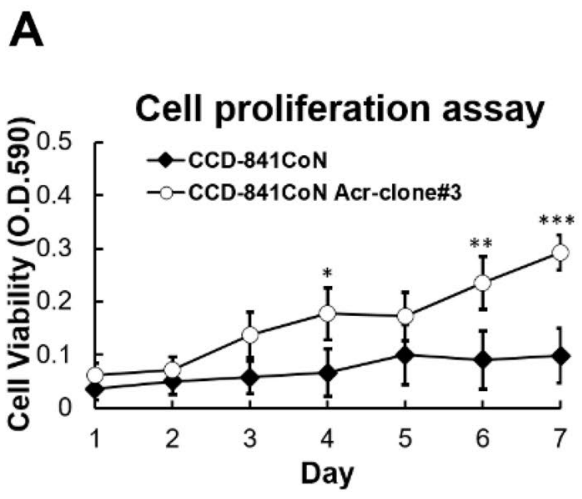

C

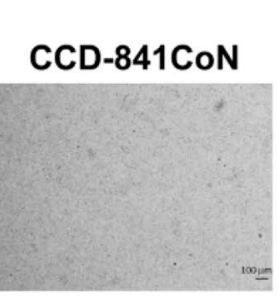

CCD-841CoN Acr-clone\#3

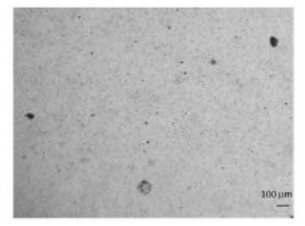

Soft agar colony formation

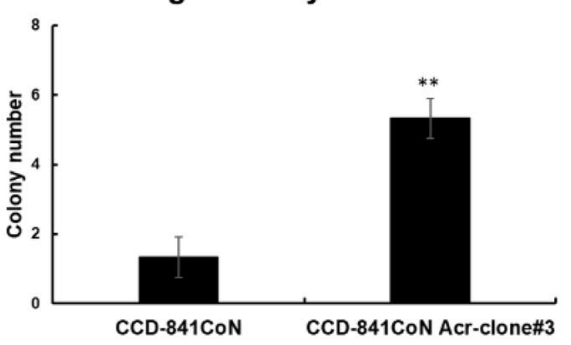

B

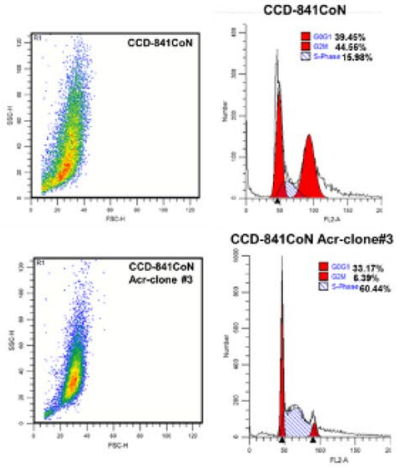

D
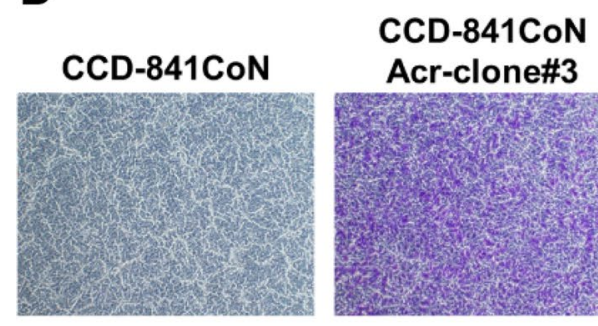
Acr-clone\#3

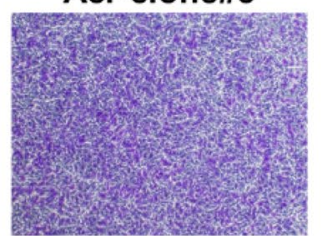

Migration assay

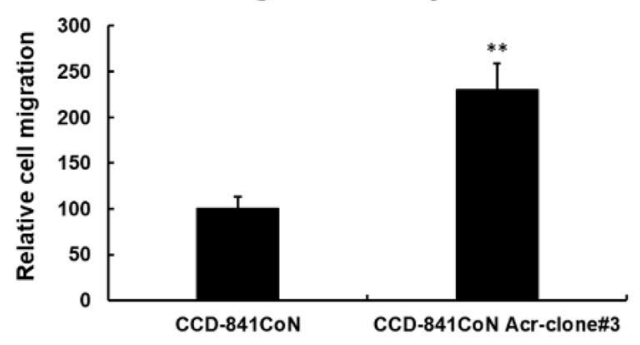

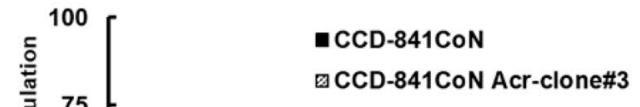

øCCD-841CoN Acr-clone\#3

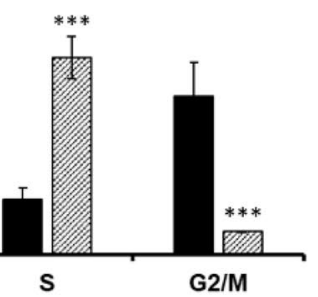

$\mathbf{E}$

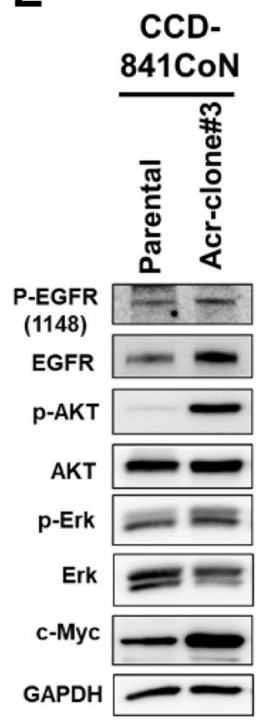

Figure 4. Acrolein induced oncogenic transformation in CCD-841CoN cells. CCD-841CoN cells were treated with acrolein $(\mathrm{Acr}, 7.5 \mu \mathrm{M})$ for one month and named CCD-841CoN Acr-clone\#3. (A) Cell proliferation of CCD-841CoN Acr-clone\#3 was analyzed using MTT assays. (B) Cell cycle progression of CCD-841CoN Acr-clone\#3 was analyzed using cell cycle analysis with PI staining. (C) Soft agar anchorage-dependent cell growth of CCD-841CoN Acr-clone\#3 was analyzed using a soft agar assay. Scale bar: $100 \mu \mathrm{m}$. (D) The cell migration activity of CCD-841CoN Acr-clone\#3 cells was analyzed using Transwell migration analysis. Student's $\mathrm{t}$ tests were used to determine statistical significance, and two-tailed $\mathrm{p}$-values are shown. ${ }^{*} \mathrm{p}<0.05,{ }^{* *} \mathrm{p}<0.01$, ${ }^{* * *} \mathrm{p}<0.005$ compared with CCD-841CoN parental cells. (E) Western blot analysis of the RAS/ERK and AKT pathways in acrolein-transformed clones compared to parental CCD-841CoN cells. Original Western blots of (E) are shown in Supplementary Fig. 3D.

In vivo exposure to acrolein in most situations is quite low, and the effects may differ from those seen at acutely toxic doses ${ }^{10,42}$. Acrolein, the most reactive $\alpha, \beta$-unsaturated aldehyde, rapidly binds to and depletes cellular nucleophiles such as glutathione and reacts with proteins and $\mathrm{DNA}^{41-43}$. This reactivity is the basis for the cytotoxicity evident in all cells exposed to high concentrations of acrolein ${ }^{44-47}$. On the other hand, acrolein inhibits cell proliferation without causing cell death at low doses ${ }^{48-50}$. Previous studies have shown that the peak concentration of daily oral acrolein exposure has been estimated at $30 \mu \mathrm{g} / \mathrm{kg} \mathrm{bw}{ }^{25}$, which is approximately $7.5 \mu \mathrm{M}$ based on a $70 \mathrm{~kg}$ adult with a $5 \mathrm{~L}$ blood volume. Therefore, we used a sublethal dose of acrolein $(7.5 \mu \mathrm{M}, \mathrm{IC10})$ to expose NIH/3T3 cells for one month to mimic in vivo conditions (Supplementary Fig. 1A). The results showed that acrolein was able to transform NIH/3T3 cells, and NIH/3T3 Acr-clones \#3, \#4 and \#6 formed more colonies than the others (Supplementary Fig. 1B). However, cell proliferation analysis showed the opposite phenomenon (Fig. 1A, Supplementary Fig. 1C). Furthermore, NIH/3T3 Acr-clone\#4 was able to form tumors, whereas no tumors were observed in mice inoculated with NIH/3T3 parental cells or NIH/3T3 Acr-clone\#3 (data not shown) using a xenograft mouse model (Fig. 2). The mechanisms underlying acrolein-induced cell transformation may be related to the ability of acrolein to deplete cellular thiols or other nucleophiles and/or to effects on gene activation, either directly or subsequent to effects on redox-regulated transcription factors ${ }^{10,42}$. To explore the possible signaling involved in acrolein-induced oncogenic transformation, we used a cDNA microarray with IPA analysis, and the results showed that the RAS/MPAK pathway was the top of canonical pathway analysis (Fig. 3A).

Previous studies have shown that alterations in EGFR-related Ras-Raf-MAPK and PI3K-Akt pathways are involved in the pathogenesis of up to $55 \%$ and $15 \%$ of CRC, respectively ${ }^{51}$. Upregulation of c-myc protein plays 
A

B

C

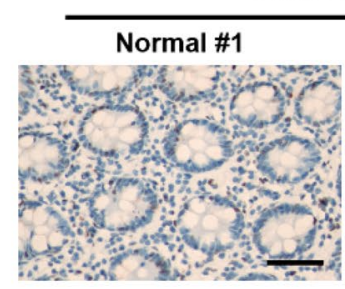

Normal \#2

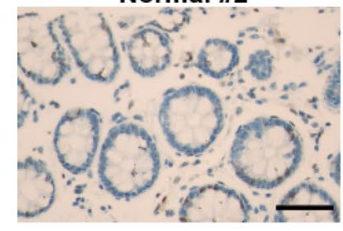

Tumor \#1

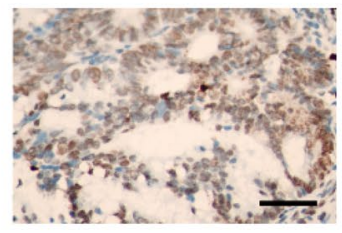

Tumor \#2

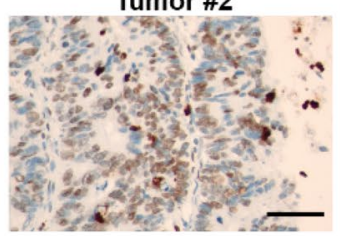

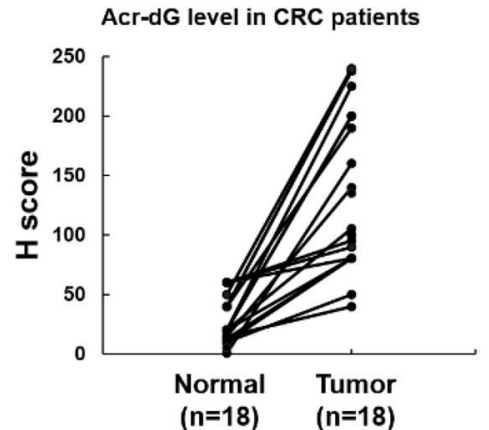

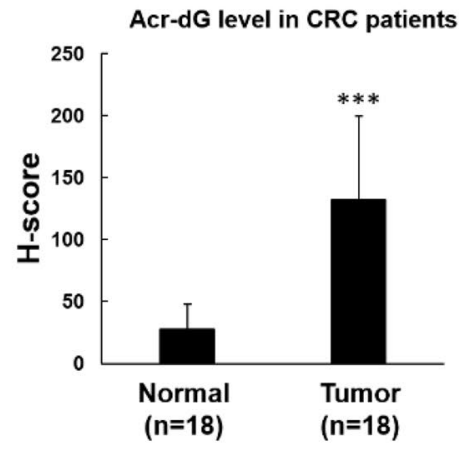

D

Strata + High Acr-dG + Low Acr-dG

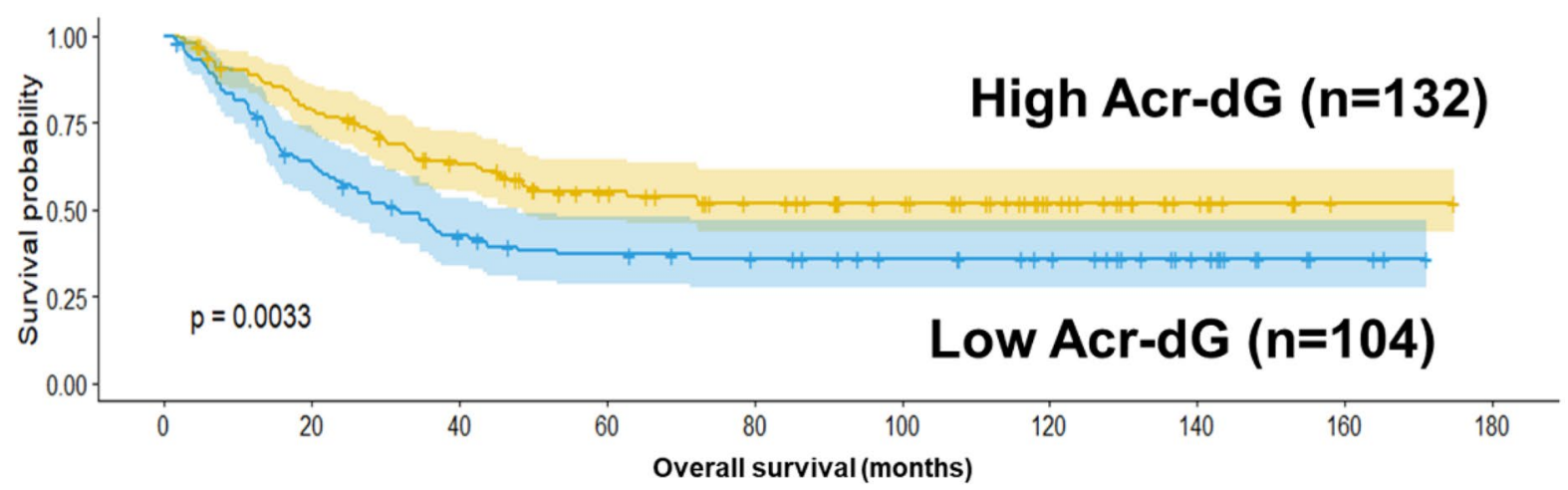

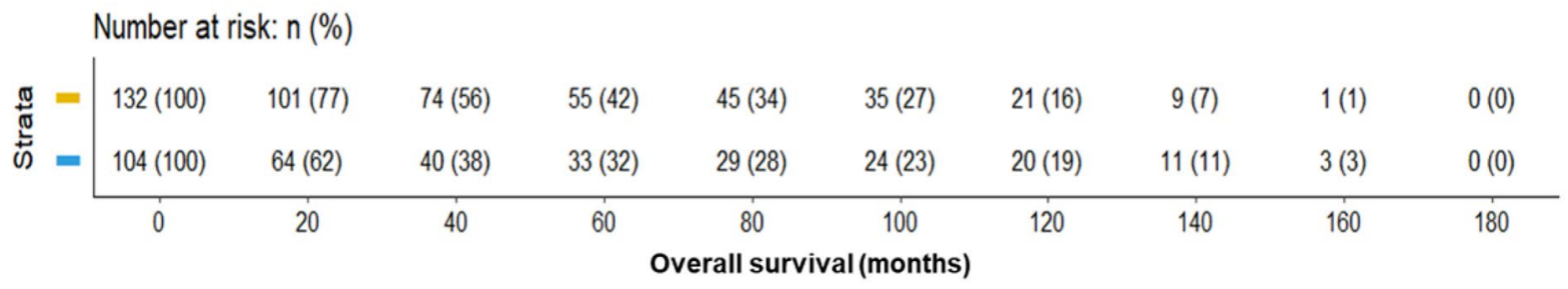

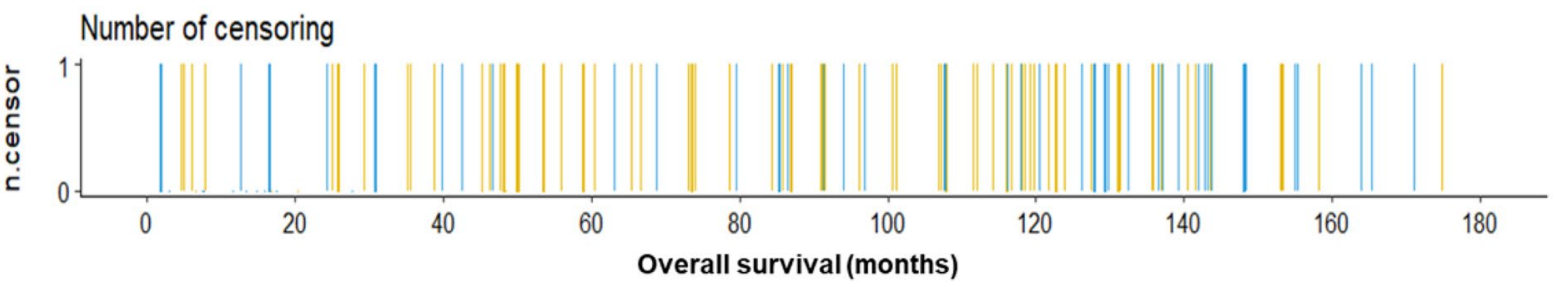

Figure 5. Immunohistochemical staining for Acr-dG adducts in eighteen CRC patients and Kaplan-Meier survival analysis of the high and low Acr-dG expression groups in CRC patients. (A) Representative image of Acr-dG adducts in normal epithelial cells adjacent to CRC tumor tissues (magnification, $\times 400$ ). Scale bar: $50 \mu \mathrm{m}$. (B,C) Quantification of Acr-dG adducts in normal epithelial cells adjacent to CRC tumor tissues. (B) Relative Acr-dG levels between normal and tumor tissues in 18 individual CRC patients. (C) Average Acr-dG levels in normal tissues and tissues from $18 \mathrm{CRC}$ patients. Student's $t$ tests were used to determine statistical significance, and two-tailed $\mathrm{p}$-values are shown. ${ }^{*} \mathrm{p}<0.05,{ }^{* *} \mathrm{p}<0.01,{ }^{* * *} \mathrm{p}<0.005$ compared between tumor tissues and normal tissues. (D) IHC analysis of Acr-dG levels in CRC tissues $(n=236)$ was performed as described in the "Materials and Methods". The Kaplan-Meier survival analysis survival plot was analyzed with the $\mathrm{R}$ software ( $\mathrm{R}$ version 4.1.0). The number at risk and the number of censors in study cases are listed. Additionally, the endpoint was cancer-specific death. 


\begin{tabular}{|c|c|c|c|c|c|c|}
\hline & & \multicolumn{4}{|c|}{ Acr-dG levels } & \multirow[b]{3}{*}{ P value } \\
\hline & & \multicolumn{2}{|c|}{ Low $($ h score $<50)$} & \multicolumn{2}{|c|}{$\begin{array}{l}\text { High }(h \\
\text { score }>=50)\end{array}$} & \\
\hline & & Count & $\%$ & Count & $\%$ & \\
\hline \multirow{3}{*}{ Age } & Mean \pm SD & \multicolumn{2}{|c|}{$64.7 \pm 13.8$} & \multicolumn{2}{|c|}{$63.8 \pm 12.6$} & \\
\hline & $<70$ years & 56 & $53.8 \%$ & 83 & $62.9 \%$ & 0.161 \\
\hline & $\geq 70$ years & 48 & $46.2 \%$ & 49 & $37.1 \%$ & 0.101 \\
\hline \multirow{2}{*}{ Sex } & Female & 40 & $38.5 \%$ & 44 & $33.3 \%$ & \multirow{2}{*}{0.414} \\
\hline & Male & 64 & $61.5 \%$ & 88 & $66.7 \%$ & \\
\hline \multirow{2}{*}{ Location } & Left & 43 & $41.3 \%$ & 60 & $45.5 \%$ & \multirow{2}{*}{0.528} \\
\hline & Right & 61 & $58.7 \%$ & 72 & $54.5 \%$ & \\
\hline \multirow{3}{*}{ Pathology } & Adenocarcinoma & 91 & $87.5 \%$ & 120 & $90.9 \%$ & \multirow{3}{*}{$<0.001^{\mathrm{a}}$} \\
\hline & Carcinoma & 1 & $1.0 \%$ & 0 & $.0 \%$ & \\
\hline & Mucinous adenocarcinoma & 12 & $11.5 \%$ & 12 & $9.1 \%$ & \\
\hline \multirow{4}{*}{ Clinical Stage (AJCC 6th) } & $\mathrm{I}$ & 6 & $5.8 \%$ & 12 & $9.1 \%$ & \multirow{4}{*}{$0.041^{*}$} \\
\hline & II & 26 & $25.0 \%$ & 41 & $31.1 \%$ & \\
\hline & III & 26 & $25.0 \%$ & 44 & $33.3 \%$ & \\
\hline & IV & 46 & $44.2 \%$ & 35 & $26.5 \%$ & \\
\hline \multirow{2}{*}{ Grade } & Low & 90 & $86.5 \%$ & 126 & $95.5 \%$ & \multirow{2}{*}{$0.015^{*}$} \\
\hline & High & 14 & $13.5 \%$ & 6 & $4.5 \%$ & \\
\hline \multirow{2}{*}{ Mucinous component } & No & 64 & $61.5 \%$ & 83 & $62.9 \%$ & \multirow{2}{*}{0.833} \\
\hline & Yes & 40 & $38.5 \%$ & 49 & $37.1 \%$ & \\
\hline \multirow{2}{*}{ LVSI } & No & 74 & $71.2 \%$ & 107 & $81.1 \%$ & \multirow{2}{*}{0.074} \\
\hline & Yes & 30 & $28.8 \%$ & 25 & $18.9 \%$ & \\
\hline \multirow{2}{*}{ PNI } & No & 37 & $88.1 \%$ & 38 & $97.4 \%$ & \multirow{2}{*}{$0.203^{\mathrm{a}}$} \\
\hline & Yes & 5 & $11.9 \%$ & 1 & $2.6 \%$ & \\
\hline \multirow{2}{*}{ Death event } & No & 40 & $38.5 \%$ & 73 & $55.3 \%$ & \multirow{2}{*}{$0.010^{*}$} \\
\hline & Yes & 64 & $61.5 \%$ & 59 & $44.7 \%$ & \\
\hline
\end{tabular}

Table 1. Clinical characteristics of CRC patients. AJCC American Joint Committee on Cancer, LVSI lymphvascular space invasion, PNI perineural invasion. ${ }^{\star} \mathrm{P}<0.05$, the chi-square statistic is significant at the 0.05 level. ${ }^{a}$ Fisher's exact test.

an essential role in tumorigenesis through frequently altered kinase MAPK and RAS pathways in CRC ${ }^{52}$. In this study, we found that acrolein upregulated the RAS/MAPK pathway followed by overexpression of c-myc in both NIH/3T3 and colon cells, CCD-841coN (Figs. 3,4). Consistently, increased c-myc expression was also observed in these CRC tumor tissues (Supplementary Fig. 5), along with higher Acr-dG adducts in these tumor tissues (Fig. 5A-C). Acrolein is a highly reactive aldehyde reacting with dG of DNA to form Acr-dG adducts, which were shown to be mutagenic ${ }^{15,31-40}$. It is unclear whether acrolein induces mutations in RAS/MAPK pathways. These results showed that acrolein may be involved in colon tumorigenesis and that the underlying mechanism is possible through activation of the RAS/MAPK pathway and upregulation of c-myc.

Interestingly, we found that CRC patients with higher Acr-dG expression in tumor tissues had a better prognosis (Fig. 5D, Table 1). A possible explanation is that Acr-dG adducts are involved in the initiation of colon tumorigenesis; however, accumulating high amounts of Acr-dG adducts trigger cellular apoptosis. Acrolein can be produced through lipid peroxidation in fast dividing cells such as cancer cells ${ }^{53}$. Our previous studies have shown that hypoxia induces acrolein production, resulting in cellular apoptosis ${ }^{54}$. Furthermore, an inverse correlation between cell viability and relative Acr-dG adduct levels was observed in acrolein-treated NIH/3T3 cells (Supplementary Fig. 8). In addition, acrolein induced cytotoxicity in the colon cancer cell lines SW480 and HCT116 (Supplementary Fig. 9). This may explain why CRC patients with higher Acr-dG adduct levels were associated with better survival. However, the detailed mechanisms still need further investigation.

The major restriction of this study is that NIH/3T3 is a mouse fibroblast cell model, and the genetic background may not be correlated with epithelia, although NIH/3T3 has been recognized as a cell line for tumorigenesis studies in vitro and in vivo ${ }^{17,55,56}$. We could not observe EGFR expression in NIH/3T3 cells, which is similar to previous studies showing that NIH/3T3 cells lack EGFR ${ }^{57,58}$. Therefore, we tried to use a normal colon cell line, CCD-841CoN, as a model and found that acrolein indeed induced activation of the RAS/MAPK pathway, which was similar to NIH/3T3 (Fig. 3E). In addition, acrolein also induced cell proliferation, colony formation activity and cell migration capacity in CCD-841CoN cells (Fig. 4A-C). Activation of the RAS/MAPK signaling pathway was also observed in the CCD-841CoN Acr-clone (Fig. 4D). Furthermore, we found that acrolein increased the phosphorylation of EGFR, indicating that activation of EGFR results in the downstream RAS/MAPK pathway in CCD-841CoN (Figs. 3E, 4D).

Taken together, we found that acrolein induced oncogenic transformation using NIH/3T3 cells in a xenograft mouse model through upregulation of the RAS/MAPK pathway. In addition, higher acrolein-induced DNA 
damage (Acr-dG adducts) was observed in tumor tissues than in adjacent normal epithelial cells in CRC patients. Interestingly, increased Acr-dG levels were associated with better prognosis in CRC patients. To our knowledge, this is the first study to show that acrolein is important in oncogenic transformation through activation of the RAS/MAPK signaling pathway, contributing to colon carcinogenesis. Thus, this study provides insight for the early detection and prevention of colon cancer in the future.

\section{Materials and methods}

Cell culture and acrolein treatment. A mouse fibroblast cell line (NIH/3T3) and human normal colorectal cell CCD 841 CoN (ATCC CRL-1790) were purchased from ATCC and maintained in Dulbecco's modified Eagle's medium (DMEM) supplemented with 10\% BCS and 15\% FBS, respectively. Acrolein stock solution (Sigma-Aldrich) was prepared freshly before use. Cells at $70 \%$ confluency were treated with different concentrations of acrolein $(0-10 \mu \mathrm{M})$ in complete culture medium for 1 months at $37^{\circ} \mathrm{C}$ in the dark, and acroleincontaining medium was changed every two days.

Cell proliferation assay. Cell proliferation was determined using modified 3-(4,5- dimethylthiazol-2-yl)2,5-diphenyl tetrazolium (MTT; Sigma, St. Louis, MO) assay ${ }^{59}$. Briefly, cells (1000/well) were seeded in 96-well plates overnight and measured every day for 7 days. The resulting formazan dissolved in DMSO was measured at $570 \mathrm{~nm}$, and the results are presented as the percentage of the control values. All of these experiments were performed in triplicate and were repeated independently at least three times.

Flow cytometry analysis of cell cycle phases. Cells were washed twice in ice-cold PBS and fixed in ice-cold $70 \%$ ethanol for $30 \mathrm{~min}$ or overnight at $4{ }^{\circ} \mathrm{C}$. Cells were then washed in PBS and digested with DNasefree RNase A ( $50 \mathrm{U} / \mathrm{mL})$ at $37^{\circ} \mathrm{C}$ for $30 \mathrm{~min}$. Before flow cytometry analysis, cells were resuspended in $500 \mu \mathrm{L}$ of propidium iodide (PI, $10 \mu \mathrm{g} / \mathrm{mL}$; Sigma) for DNA staining. PI staining was used to measure the cell cycle status using a Becton-Dickinson FACScan instrument and Cell Quest software.

Soft agar colony formation assay. A soft agar colony formation assay was performed as described previously ${ }^{60}$. Briefly, a 3-mL aliquot of $1.2 \%$ agar in culture medium was plated in $60-\mathrm{mm}$ dishes. Then, 1,000 transformed malignant or untransformed cells were mixed with $3 \mathrm{~mL}$ of $0.35 \%$ agar in medium and plated on solidified bottom agar. When the top agar solidified, the dishes were transferred to an incubator and cultured for 30 days. Two or three drops of the medium were added to each dish three times a week. After culturing for 30 days, the visible cell colonies were photographed and counted.

Tumor sphere culture assay. Acrolein-transformed NIH/3T3 clones were trypsinized and resuspended at 1000 cells/Ultra-Low Attachment 96-well Plate (Corning) in culture medium containing $2 \mathrm{mM}$ L-glutamine, N2 supplement, B27 supplement, $20 \mathrm{ng} / \mathrm{mL}$ hrEGF (Sigma), and $20 \mathrm{ng} / \mathrm{mL}$ hrbFGF (Sigma) for two weeks. Fresh growth factors were added to the cells twice a week. Cumulative total numbers of cells from the spheroid cultures were calculated.

Cell migration assay. The cell migration assay was performed in vitro utilizing modified Boyden chambers with a Transwell apparatus (polycarbonate membranes with $8-\mathrm{mm}$ pores, Corning) ${ }^{61}$. Parental NIH/3T3 or $\mathrm{NIH} / 3 \mathrm{~T} 3 \mathrm{Acr}$ clones $\left(5 \times 10^{4}\right.$ in $500 \mu \mathrm{L}$ of growth medium/well, 6-well plates) were added to the upper chamber, and the lower chamber contained $750 \mu \mathrm{L}$ of growth medium supplemented with $10 \%$ FCS. Cells on the upper membrane surface were wiped with a cotton swab after $24 \mathrm{~h}$ of incubation at $37^{\circ} \mathrm{C}$ in a $5 \% \mathrm{CO}_{2}$ incubator. Membranes were then fixed and stained with crystal violet, and cells that migrated to the lower membrane surface were counted in nine random fields using a microscope at $200 \times$ magnification. These experiments were performed in triplicate and were repeated at least three times.

Immunoblotting analysis. Cells were washed twice with ice-cold PBS and lysed on ice for $20 \mathrm{~min}$ in radioimmunoprecipitation assay (RIPA) lysis buffer $(20 \mathrm{mM}$ Tris $\mathrm{HCl}, 150 \mathrm{mM} \mathrm{NaCl}, 1 \%$ (v/v) NP-40, $1 \%$ (w/v) sodium deoxycholate, $1 \mathrm{mM}$ ethylenediaminetetraacetate (EDTA), $0.1 \%$ (w/v) sodium dodecyl sulfate polyacrylamide (SDS) plus protease and phosphatase inhibitors). Lysates were then centrifuged at 13,200 rpm for $10 \mathrm{~min}$, and the protein concentrations of supernatant were determined by a BCA Protein Assay Kit. Protein samples $(30 \mu \mathrm{g})$ were run on $8-100 \%$ SDS-polyacrylamide gel electrophoresis and then transferred into polyvinylidene difluoride (Bio-Rad, U.S.A.) at $90 \mathrm{~V}$ for $120 \mathrm{~min}$. Proteins were transferred onto nitrocellulose membranes (Bio-Rad). Blots were probed with primary antibodies overnight at $4{ }^{\circ} \mathrm{C}$. Primary antibodies included: P-EGFR (Tyr1148, 1:1000, Cell Signaling \#4404); EGFR (1:1000, Cell Signaling \#2232); RAS (1:1000, Cell Signaling \#3965); p-AKT (1:1000, Cell Signaling \#4058); AKT (1:1000, Cell Signaling \#4685); P-p44/42 MAPK (Erk1/2) (Thr202/Tyr204) (1:1000, Cell Signaling \#9101); p44/42 MAPK (Erk1/2) (Thr202/Tyr204) antibody \#9102; Cyclin D1 (1:1000, Cell Signaling \#2978); c-myc (1:500, Santa Cruz, sc-42) and anti-acrolein antibody [10A10] (1:1000, Abcam, ab240918)) for acrolein-protein conjugates (Acr-PC). After primary antibody incubation, the membrane was washed and incubated with horseradish peroxidase-conjugated secondary IgG (1:3000; Millipore) for $1 \mathrm{~h}$ at room temperature. Immunoreactive bands were detected using Amersham Enhanced Chemiluminescence (Amersham Pharmacia Biotech, Piscataway, NJ, U.S.A.). The bound primary and secondary antibodies were stripped by incubating the membrane in stripping buffer (100 mM 2-mercaptoethanol, $2 \%$ SDS) for $30 \mathrm{~min}$ at room temperature. The membrane was then reprobed with GAPDH (1:1000, Cell Signaling, \#5174). 
Slot blot assay for Acr-dG detection. Analysis of Acr-dG adducts in DNA samples was based on previously described methods ${ }^{12,62}$. Briefly, buccal DNA $(0.25 \mu \mathrm{g})$ was loaded onto PVDF membranes using a Bio-Dot SF microfiltration apparatus (Bio-Rad, Hercules, CA). WesternDot 625 Western blotting kits (Invitrogen) were used for Western blot analysis according to the manufacturer's instructions. The membrane was probed overnight at $4{ }^{\circ} \mathrm{C}$ with anti-Acr-dG mouse monoclonal antibodies ${ }^{63}$. Acr-dG adducts were detected using a UVP BioDoc-It imaging system, and band density was quantified with UVP imaging software. Relative Acr-dG levels were calculated by the fluorescence intensity of Acr-dG stained with an anti-Acr-dG antibody normalized to the amount of loaded DNA stained with methylene blue.

Xenograft mouse model. Fifteen 6-week-old male Balb/c nude mice weighing 25-30 g were used. All animal experiments were approved by the Institutional Animal Care and Use Committee of National YangMing University, and the study was carried out in compliance with the ARRIVE guidelines (IACUC\#1070208rr). Tumors were induced by injecting acrolein-transformed NIH/3T3 cells $\left(5 \times 10^{6}\right.$ in $100 \mu \mathrm{l}$ of PBS per animal) subcutaneously into the right axillary fossa of mice as described previously with slight modification ${ }^{61}$. To generate the tumor growth curve, measurement of tumors was performed twice a week with a digital caliper, and volumes were calculated by (length $\mathrm{x}$ width $\left.{ }^{2}\right) / 2$. Body weight was also evaluated twice weekly. Tumor samples were collected after sacrifice. Each sample was cut in half; one half was saved in $10 \%$ formaldehyde, and one half was stored at $-80^{\circ} \mathrm{C}$ until further use.

RNA isolation and cDNA microarray analysis. Total RNA was isolated from TRIzol Reagent cells (Thermo Fisher Scientific) according to the manufacturer's instructions. RNA samples were quantified using an ND-1000 spectrophotometer (NanoDrop Technologies, Wilmington, USA) The quality was determined using an Agilent 2100 Bioanalyzer with a Nanochip (Agilent, Santa Clara, CA) following the manufacturer's instructions. Microarray hybridizations were performed using total RNA prepared from NIH/3T3 and NIH/3T3 Acr-clone\#4 cells as described previously ${ }^{48}$. GeneChip Mouse Genome 4302.0 Affymetrix oligonucleotide gene chips (Affymetrix) were analyzed at the Microarray \& Gene Expression Analysis Core Facility (VYM Genome Research Center, National Yang-Ming University) according to the Affymetrix protocols. Microarray datasets were analyzed using Ingenuity Pathway Analysis (IPA version 62089861) (QIAGEN), and bioinformatic analysis of the differentially expressed genes (DEGs, fold change $\geq 2$ ) was performed. The IPA identified biological functions that were most significant to the data set. DEGs that were associated with biological functions in the Ingenuity Knowledge Base (Ingenuity Systems) were used for the analysis. Fisher's exact test was used to calculate a $\mathrm{p}$ value that determined the probability that each biological function assigned to that network or to the data set was due to chance alone.

Collection of tissue microarray of CRC patients. A total of 236 patients diagnosed with CRC at Taipei Veterans General Hospital were enrolled. Disease stage was assessed based on the American Joint Committee on Cancer staging system, 6th edition. Clinicopathological staging and clinical course were determined by searching a computer database containing detailed information. The medical residual samples of the patients were acquired from the residual sample bank of Taipei Veterans General Hospital, and this study was approved by the Institutional Review Board of Taipei Veterans General Hospital (VGHIRB, IRB\#2020-01-010BC). VGHIRB waived the requirement for the use of informed consent. Patients were classified based on their primary tumor locations, including the right-sided colon (tumors originating in the cecum, ascending colon, hepatic flexure, and transverse colon), left-sided colorectum (tumors originating in the splenic flexure, descending colon, sigmoid colon, rectosigmoid junction, and rectum. Low-grade cancers have cancer cells that are well differentiated or moderately differentiated. High-grade cancers have cancer cells that are poorly differentiated or undifferentiated.

Immunohistochemistry (IHC) analysis of the Acr-dG adduct and c-myc. For the tissue microarray (TMA), hematoxylin and eosin-stained sections from each paraffin-embedded, formalin-fixed block were used to define diagnostic areas, and a representative $0.6 \mathrm{~mm}$ core was obtained from each case and inserted in a grid pattern into a recipient paraffin block ${ }^{49,50}$. IHC analysis was carried out as previously described with slight modification $^{64}$. Briefly, sections $(4 \mu \mathrm{m})$ were then deparaffinized in xylene and rehydrated in a descending ethanol series. To enhance immunoreactivity, sections were incubated in Tris-EDTA, pH 6.0, and boiled for $12 \mathrm{~min}$. Endogenous peroxidase activity was eliminated by incubation in hydrogen peroxide. Incubation with primary antibodies for Acr-dG antibody (generated in house) and c-myc (Santa Cruz, sc-40) was performed overnight at $4{ }^{\circ} \mathrm{C}$ in $1 \%$ BSA in phosphate-buffered saline (PBS). Bound antibodies were visualized with DAB (diaminobenzidine) used as a chromogen, and omission of the primary antibody served as a negative control. Positive controls (normal liver) were stained in parallel with each set of TMAs studied. Assessment of Acr-dG and c-myc immunoexpression was performed by light microscopy at $\times 400$ magnification by a pathologist.

Statistical analyses. Descriptive statistics are presented as the mean \pm standard deviation or as the number (percentage). Student's t tests were used to determine statistical significance, and two-tailed P-values are shown. A minimum of three independent replicate experiments was performed to justify the use of statistical tests. Survival was analyzed using Kaplan-Meier survival analysis, and the log rank test was used for comparison between the two groups. Multivariate analysis was performed using chi-square analysis or Fisher's exact test. Statistical significance was defined as a $p<0.05$. All analyses were performed with the IBM SPSS Statistics software package, version 23.0 or $\mathrm{R}$ software ( $\mathrm{R}$ version 4.1.0). 
Ethics approval. Our study protocol was approved by the Institutional Review Board of Taipei Veterans General Hospital (IRB\#2020-01-010BC) and the study was carried out in accordance with the Declaration of Helsinki principles. All animal experiments were approved by the Institutional Animal Care and Use Committee of National Yang-Ming University and was carried out in compliance with the ARRIVE guidelines (IACUC\#1070208rr).

Received: 7 January 2021; Accepted: 1 June 2021

Published online: 15 June 2021

\section{References}

1. Howlader, N. N. A., Krapcho, M. et al. SEER Cancer Statistics Review, 1975-2013. http://seer.cancer.gov/csr/1975_2013/.Googl eScholar (2016).

2. Waliszewski, P. Controversies about the genetic model of colorectal tumorigenesis. Pol. J. Pathol. 46, 239-243 (1995).

3. Fearon, E. R. \& Vogelstein, B. A genetic model for colorectal tumorigenesis. Cell 61, 759-767. https://doi.org/10.1016/00928674(90)90186-i (1990).

4. Tiwari, A., Saraf, S., Verma, A., Panda, P. K. \& Jain, S. K. Novel targeting approaches and signaling pathways of colorectal cancer: An insight. World J. Gastroenterol. 24, 4428-4435. https://doi.org/10.3748/wjg.v24.i39.4428 (2018).

5. Farooqi, A. A., de la Roche, M., Djamgoz, M. B. A. \& Siddik, Z. H. Overview of the oncogenic signaling pathways in colorectal cancer: Mechanistic insights. Semin. Cancer Biol. 58, 65-79. https://doi.org/10.1016/j.semcancer.2019.01.001 (2019).

6. Walther, A. et al. Genetic prognostic and predictive markers in colorectal cancer. Nat. Rev. Cancer 9, 489-499. https://doi.org/10. $1038 / \mathrm{nrc2645}(2009)$

7. Vargas, A. J. \& Thompson, P. A. Diet and nutrient factors in colorectal cancer risk. Nutr. Clin. Pract. 27, 613-623. https://doi.org/ 10.1177/0884533612454885 (2012).

8. Nystrom, M. \& Mutanen, M. Diet and epigenetics in colon cancer. World J. Gastroenterol. 15, 257-263. https://doi.org/10.3748/ wjg.15.257 (2009).

9. Newmark, H. L. et al. A western-style diet induces benign and malignant neoplasms in the colon of normal C57Bl/6 mice. Carcinogenesis 22, 1871-1875. https://doi.org/10.1093/carcin/22.11.1871 (2001).

10. Moghe, A. et al. Molecular mechanisms of acrolein toxicity: Relevance to human disease. Toxicol. Sci. 143, 242-255. https://doi. org/10.1093/toxsci/kfu233 (2015).

11. Bein, K. \& Leikauf, G. D. Acrolein-A pulmonary hazard. Mol. Nutr. Food Res. 55, 1342-1360. https://doi.org/10.1002/mnfr.20110 0279 (2011).

12. Tsou, H. H. et al. Acrolein is involved in the synergistic potential of cigarette smoking- and betel quid chewing-related human oral cancer. Cancer Epidemiol. Biomark. Prevent. 28, 954-962. https://doi.org/10.1158/1055-9965.EPI-18-1033 (2019).

13. Lee, H. W. et al. Cigarette side-stream smoke lung and bladder carcinogenesis: Inducing mutagenic acrolein-DNA adducts, inhibiting DNA repair and enhancing anchorage-independent-growth cell transformation. Oncotarget 6, 33226-33236. https://doi.org/ 10.18632/oncotarget.5429 (2015).

14. Zhang, C. et al. Structural resilience of the gut microbiota in adult mice under high-fat dietary perturbations. ISME J. 6, $1848-1857$. https://doi.org/10.1038/ismej.2012.27 (2012).

15. Tang, M. S. et al. Acrolein induced DNA damage, mutagenicity and effect on DNA repair. Mol. Nutr. Food Res. 55, $1291-1300$. https://doi.org/10.1002/mnfr.201100148 (2011).

16. Feng, Z., Hu, W., Hu, Y. \& Tang, M. S. Acrolein is a major cigarette-related lung cancer agent: Preferential binding at p53 mutational hotspots and inhibition of DNA repair. Proc. Natl. Acad. Sci. U S A 103, 15404-15409. https://doi.org/10.1073/pnas.0607031103 (2006).

17. Greig, R. G. et al. Tumorigenic and metastatic properties of "normal" and ras-transfected NIH/3T3 cells. Proc. Natl. Acad. Sci. U S A 82, 3698-3701. https://doi.org/10.1073/pnas.82.11.3698 (1985).

18. Richard, H., Stadler, D. R. L. Process-Induced Food Toxicants: Occurrence, Formation, Mitigation, and Health Risks. 51-74 (Wiley, 2008).

19. Ferretti A, F. V. Lactose casein (Maillard) browning system: Volatile components. J. Agric. Food. 19, 5 (1971).

20. Alarcon, R. A. Formation of acrolein from various amino-acids and polyamines under degradation at 100 degrees C. Environ. Res. 12, 317-326 (1976).

21. Lee, Y. \& Sayre, L. M. Reaffirmation that metabolism of polyamines by bovine plasma amine oxidase occurs strictly at the primary amino termini. J. Biol. Chem. 273, 19490-19494 (1998).

22. Osorio, V. M. \& de Lourdes Cardeal, Z. Determination of acrolein in French fries by solid-phase microextraction gas chromatography and mass spectrometry. J. Chromatogr. A 1218, 3332-3336. https://doi.org/10.1016/j.chroma.2010.11.068 (2011).

23. Zhang, J., Sturla, S., Lacroix, C. \& Schwab, C. Gut microbial glycerol metabolism as an endogenous acrolein source. MBio 9. https:// doi.org/10.1128/mBio.01947-17 (2018).

24. Wang, T. W., Liu, J. H., Tsou, H. H., Liu, T. Y. \& Wang, H. T. Identification of acrolein metabolites in human buccal cells, blood, and urine after consumption of commercial fried food. Food Sci. Nutr. 7, 1668-1676. https://doi.org/10.1002/fsn3.1001 (2019).

25. Abraham, K. et al. Toxicology and risk assessment of acrolein in food. Mol. Nutr. Food Res. 55, 1277-1290. https://doi.org/10.1002/ mnfr.201100481 (2011).

26. Stevens, J. F. \& Maier, C. S. Acrolein: Sources, metabolism, and biomolecular interactions relevant to human health and disease. Mol. Nutr. Food Res. 52, 7-25 (2008).

27. Meyerhardt, J. A. et al. Association of dietary patterns with cancer recurrence and survival in patients with stage III colon cancer. JAMA 298, 754-764. https://doi.org/10.1001/jama.298.7.754 (2007).

28. Faivre, J., Bouvier, A. M. \& Bonithon-Kopp, C. Epidemiology and screening of colorectal cancer. Best Pract. Res. Clin. Gastroenterol. 16, 187-199. https://doi.org/10.1053/bega.2001.0280 (2002).

29. Boyle, P. \& Leon, M. E. Epidemiology of colorectal cancer. Br. Med. Bull. 64, 1-25. https://doi.org/10.1093/bmb/64.1.1 (2002).

30. Chung, F. L., Young, R. \& Hecht, S. S. Formation of cyclic 1, N2-propanodeoxyguanosine adducts in DNA upon reaction with acrolein or crotonaldehyde. Can. Res. 44, 990-995 (1984).

31. Wang, H.-T. Effect of Acrolein in DNA Damage, DNA Repair and Lung Carcinogenesis. Ph.D. thesis (New York University, 2012).

32. Wang, H. T., Zhang, S., Hu, Y. \& Tang, M. S. Mutagenicity and sequence specificity of acrolein-DNA adducts. Chem. Res. Toxicol. 22, 511-517. https://doi.org/10.1021/tx800369y (2009).

33. Yang, I. Y. et al. Mammalian translesion DNA synthesis across an acrolein-derived deoxyguanosine adduct. Participation of DNA polymerase eta in error-prone synthesis in human cells. J. Biol. Chem. 278, 13989-13994. https://doi.org/10.1074/jbc.M212535200 (2003). 
34. Sanchez, A. M. et al. Comparative evaluation of the bioreactivity and mutagenic spectra of acrolein-derived alpha-HOPdG and gamma-HOPdG regioisomeric deoxyguanosine adducts. Chem. Res. Toxicol. 16, 1019-1028 (2003).

35. Minko, I. G. et al. Translesion synthesis past acrolein-derived DNA adduct, gamma -hydroxypropanodeoxyguanosine, by yeast and human DNA polymerase eta. J. Biol. Chem. 278, 784-790. https://doi.org/10.1074/jbc.M207774200M207774200 (2003).

36. Yang, I. Y. et al. Mutagenesis by acrolein-derived propanodeoxyguanosine adducts in human cells. Biochemistry 41, 13826-13832 (2002).

37. Kanuri, M. et al. Error prone translesion synthesis past gamma-hydroxypropano deoxyguanosine, the primary acrolein-derived adduct in mammalian cells. J. Biol. Chem. 277, 18257-18265 (2002).

38. Yang, I. Y. et al. Responses to the major acrolein-derived deoxyguanosine adduct in Escherichia coli. J. Biol. Chem. 276, 9071-9076 (2001).

39. VanderVeen, L. A. et al. Evaluation of the mutagenic potential of the principal DNA adduct of acrolein. J. Biol. Chem. 276, 9066-9070 (2001).

40. Kawanishi, M. et al. Molecular analysis of mutations induced by acrolein in human fibroblast cells using supF shuttle vector plasmids. Mutat. Res. 417, 65-73 (1998).

41. LoPachin, R. M., Gavin, T., Petersen, D. R. \& Barber, D. S. Molecular mechanisms of 4-hydroxy-2-nonenal and acrolein toxicity: nucleophilic targets and adduct formation. Chem. Res. Toxicol. 22, 1499-1508. https://doi.org/10.1021/tx900147g (2009).

42. Kehrer, J. P. \& Biswal, S. S. The molecular effects of acrolein. Toxicol. Sci. 57, 6-15 (2000).

43. Cai, J., Bhatnagar, A. \& Pierce, W. M. Jr. Protein modification by acrolein: Formation and stability of cysteine adducts. Chem. Res. Toxicol. 22, 708-716. https://doi.org/10.1021/tx800465m (2009).

44. Kachel, D. L. \& Martin, W. J., 2nd. Cyclophosphamide-induced lung toxicity: Mechanism of endothelial cell injury. J. Pharmacol. Exp. Ther. 268, 42-46 (1994).

45. Patel, J. M. \& Block, E. R. Acrolein-induced injury to cultured pulmonary artery endothelial cells. Toxicol. Appl. Pharmacol. 122, 46-53. https://doi.org/10.1006/taap.1993.1170 (1993).

46. Wang, H. T. et al. Acrolein induces mtDNA damages, mitochondrial fission and mitophagy in human lung cells. Oncotarget 8, 70406-70421. https://doi.org/10.18632/oncotarget.19710 (2017).

47. Huang, C. H., Chen, Y. T., Lin, J. H. \& Wang, H. T. Acrolein induces ribotoxic stress in human cancer cells regardless of p53 status. Toxicol. In Vitro 52, 265-271. https://doi.org/10.1016/j.tiv.2018.06.022 (2018).

48. Tsai, W. C. et al. MicroRNA-122 plays a critical role in liver homeostasis and hepatocarcinogenesis. J. Clin. Investig. 122, $2884-2897$. https://doi.org/10.1172/JCI63455 (2012).

49. Voduc, D., Kenney, C. \& Nielsen, T. O. Tissue microarrays in clinical oncology. Semin. Radiat. Oncol. 18, 89-97. https://doi.org/ 10.1016/j.semradonc.2007.10.006 (2008).

50. Kallioniemi, O. P., Wagner, U., Kononen, J. \& Sauter, G. Tissue microarray technology for high-throughput molecular profiling of cancer. Hum. Mol. Genet. 10, 657-662. https://doi.org/10.1093/hmg/10.7.657 (2001).

51. Cancer Genome Atlas, N. Comprehensive molecular characterization of human colon and rectal cancer. Nature 487, 330-337. https://doi.org/10.1038/nature11252 (2012).

52. Strippoli, A. et al. c-MYC expression is a possible keystone in the colorectal cancer resistance to EGFR inhibitors. Cancers 12. https://doi.org/10.3390/cancers12030638 (2020).

53. Esterbauer, H., Schaur, R. J. \& Zollner, H. Chemistry and biochemistry of 4-hydroxynonenal, malonaldehyde and related aldehydes. Free Radic. Biol. Med. 11, 81-128. https://doi.org/10.1016/0891-5849(91)90192-6 (1991).

54. Liu, J. H. et al. Acrolein is involved in ischemic stroke-induced neurotoxicity through spermidine/spermine-N1-acetyltransferase activation. Exp. Neurol. 323, 113066. https://doi.org/10.1016/j.expneurol.2019.113066 (2020).

55. Shimizu, K. et al. Three human transforming genes are related to the viral ras oncogenes. Proc. Natl. Acad. Sci. U S A 80, 2112-2116. https://doi.org/10.1073/pnas.80.8.2112 (1983).

56. Perucho, M. et al. Human-tumor-derived cell lines contain common and different transforming genes. Cell 27, 467-476. https:// doi.org/10.1016/0092-8674(81)90388-3 (1981).

57. Ju, W. D., Velu, T. J., Vass, W. C., Papageorge, A. G. \& Lowy, D. R. Tumorigenic transformation of NIH 3T3 cells by the autocrine synthesis of transforming growth factor alpha. New Biol. 3, 380-388 (1991).

58. Xu, H. et al. Epidermal growth factor receptor (EGFR)-related protein inhibits multiple members of the EGFR family in colon and breast cancer cells. Mol. Cancer Ther. 4, 435-442. https://doi.org/10.1158/1535-7163.MCT-04-0280 (2005).

59. Wang, H. T., Chen, T. Y., Weng, C. W., Yang, C. H. \& Tang, M. S. Acrolein preferentially damages nucleolus eliciting ribosomal stress and apoptosis in human cancer cells. Oncotarget https://doi.org/10.18632/oncotarget.12608 (2016).

60. Borowicz, S. et al. The soft agar colony formation assay. J. Vis. Exp. JoVE. e51998. https://doi.org/10.3791/51998 (2014).

61. Rao, W. et al. OVA66, a tumor associated protein, induces oncogenic transformation of NIH3T3 cells. PLoS ONE 9, e85705. https:// doi.org/10.1371/journal.pone.0085705 (2014).

62. Wang, H. T. et al. Effect of CpG methylation at different sequence context on acrolein- and BPDE-DNA binding and mutagenesis. Carcinogenesis 34, 220-227. https://doi.org/10.1093/carcin/bgs323 (2013).

63. Pan, J. et al. Detection of acrolein-derived cyclic DNA adducts in human cells by monoclonal antibodies. Chem. Res. Toxicol. 25, 2788-2795. https://doi.org/10.1021/tx3004104 (2012).

64. Vaarala, M. H., Vaisanen, M. R. \& Ristimaki, A. CIP2A expression is increased in prostate cancer. J. Exp. Clin. Cancer Res. CR 29, 136. https://doi.org/10.1186/1756-9966-29-136 (2010).

\section{Acknowledgements}

This work was funded by grants from Ministry of Science and Technology, Taiwan [109-2320-B-010-018 (H-T Wang), 109-2314-B-075-081-MY3 (H-W Teng)], Taipei Veterans General Hospital [110DHA0100397, V110C189, V109C-151 (H-W Teng)] and Chang-Gung Memorial Hospital [CMRPG3K1441 (H-C Tsai)]. The authors would like to acknowledge the support by the Biobank of Taipei Veterans General Hospital and the technical services provided by Microarray \& Gene Expression Analysis Core Facility of the National Yang-Ming University VGH Genome Research Center (VYMGC). The Gene Expression Analysis Core Facility is supported by National Research Program for Genomic Medicine (NRPGM), National Science Council.

\section{Author contributions}

H.-C.T., H.-H.T., S.-C.C., H.-W.C., H.-T.W. performed experiments; C.-C.L., W.-S.C., J.-K.J., S.-H.Y., S.-C.C., H.-W.T. collected and analyzed clinical samples. T.-Y.L., H.-W.T., H.-T.W. designed experiments and participated in manuscript writing.

\section{Competing interests}

The authors declare no competing interests. 


\section{Additional information}

Supplementary Information The online version contains supplementary material available at https://doi.org/ 10.1038/s41598-021-92035-z.

Correspondence and requests for materials should be addressed to H.-W.T. or H.-T.W.

Reprints and permissions information is available at www.nature.com/reprints.

Publisher's note Springer Nature remains neutral with regard to jurisdictional claims in published maps and institutional affiliations.

(c) (i) Open Access This article is licensed under a Creative Commons Attribution 4.0 International License, which permits use, sharing, adaptation, distribution and reproduction in any medium or format, as long as you give appropriate credit to the original author(s) and the source, provide a link to the Creative Commons licence, and indicate if changes were made. The images or other third party material in this article are included in the article's Creative Commons licence, unless indicated otherwise in a credit line to the material. If material is not included in the article's Creative Commons licence and your intended use is not permitted by statutory regulation or exceeds the permitted use, you will need to obtain permission directly from the copyright holder. To view a copy of this licence, visit http://creativecommons.org/licenses/by/4.0/.

(C) The Author(s) 2021, corrected publication 2021 